\title{
ESTANDO EM CRISE HIPERTENSIVA NUMA EMERGÊNCIA HOSPITALAR*
}

\author{
Miriam Susskind Borenstein** \\ Alacoque Lorenzini Erdmann **
}

\begin{abstract}
RESUMO: O estudo aborda a situação de saúde do cliente que apresenta crise hipertensiva ao chegarnuma emergência hospitalar. Explora dados relativos às características pessoaise clínico epidemiológicasgerais, alémdehábitosdevida quenosdãoumanoçãodaamplitudedaproblemática de estar com a saúde "emestado de emergência". Finalizando, as autoras tecem atgumas considerações sobre a necessidade de ultrapassar o foco somente biológico no atendimento a clientes com crise hipertensiva, buscandonovos hábitos saudáveis de vida.
\end{abstract}

\begin{abstract}
Thisstudy is about the health situation of the client that presents hypertensive crisis upon arriving in a hospital's emergency. It searches some data related to personal and clinical characteristics-general epidemiology, besides daily habits which give us an idea about the problematical extend of being "in an emergency situation".
\end{abstract}

UNITERMOS: Crise Hipertensiva - Emergência hospitalar - Hipertensão.

\section{INTRODUÇÃO}

Chegaraumaemergênciahospitalarpareceserum momentoespecialnavidadaspessoasque passampor este tipo de enfrentamento devido a uma crise hipertensiva(C.H.). Geralmentequandose apresenta uma crise hipertensiva, é a emergência hospitalar a portade entrada para a resoluçãodeste tipodesituação saúdedoença.

Segundo FERGUNSON e VLASSES ${ }^{4}$, a C.H. caracteriza-se como uma situação em que há uma severa elevação da pressão arterial (P.A.), sem causar no entanto nenhum dano em órgãos considerados alvo, mas que precisa ser tratada dentro de 24 horas, a fim de reduziro potencial de risco ao paciente.

Para RIBEIRO ${ }^{6}$, o quadro clínico de C.H. caracteriza-se como uma significativa elevaçãoda P.A. em um curto espaço de tempo, que requer uma terapêutica clínica emergêncial dado a eminência de ocorrerfalência cardiaca, cerebrale/ ou renal (órgãos considerados alvo). BRAUNWALD² caracteriza a crise hipertensiva quando a P.A. diastólica se apresenta acima de $130 \mathrm{mmHg}$.

Apesar de as questões semânticas ou numéricasserem estabelecidas, VIDT e GIFFORD ${ }^{7}$ afirmam que uma crise hipertensiva nunca deve serdeterminada somentetendo porbase os níveis pressóricos. O autor cita como exemplo um paciente que apresenta $240 \times 140 \mathrm{mmHg}$ de P.A., considerada elevada, mas não uma emergência, por não existir quadro de cefaléia severa ou encefalopatia, descompensação cardíaca, alteração da função renal, e portanto, sem evidência de deterioração rápida da função de órgãos alvo. Entretanto, outro paciente commoderada elevação de P.A., complicada por dissecção da aorta ou por edema agudo pulmonar, é uma verdadeira emergência hipertensiva e necessita ser tratada prontamente.

Entretanto, o indivíduo com C.H. não pode ser vistosomente pela meraquestão biológica, pois o ser humano é muito mais complexo, é um ser holístico, com necessidades de saúde que extrapolam a dimensão biológica. É um ser

\footnotetext{
Trabalho apresentado como Tema Livre no $45^{\circ}$ Congresso Brasileiro de Enfermagem. Olinda - Recife, 28 de novembro a 3 de dezembro de 1993.

** Professoras do Departamento de Enfermagem da Universidade Federal de Santa Catarina.
} 
portador de temores, ansiedade e angústia diantedo quadro que apresenta, necessitandoalém deterapêutica medicamentosa, também de uma assistência emocional.Dessemodo, acrisehipertensivageralmente vem acompanhada de uma desestruturação no eu pessoal, familiar, profissional e ambiental desseser humano. Desestruturação esta, queabalao seumundo interior/exteriorepõeemquestionamentoseusplanos devida.

Conseqüentemente, no cuidadodo ser humano com C.H., devem ser considerados todos os fatores, além do biológico, como sociais, psicológicos, culturais, econômicos e espirituais, que concorram para o restabelecimento do quadro.

Em busca de uma melhor qualidade de vida, que inclui a melhora do quadro hipertensivo, esse serhumano parece ter por expectativa um cuidado mais complexo e, portanto, mais eficiente e eficaz, cuidado este, que não se reduz simplesmente à terapêutica medicamentosa ou ao cuidado dito biológico.

O enfermeiro, ao tomar consciência desta expectativa, tem diante de si um grande desafio que perpassa por uma formação abrangente a nivel pessoal / profissional, capacitando-o a estabelecer uma relação harmoniosa com seu próprio ser e com os demais seres humanos.

Neste estudo nos propomos portanto, a investigar junto aos clientes com crise hipertensiva que procuraram a emergência hospitalar, os fatores desencadeantes desta crise, assim como um pouco de sua história de vida.

\section{METODOLOGIA}

Este estudo exploratório descritivo foi realizado no Setor deEmergência doHospital Universitário (H.U.) da Universidade Federal de Santa Catarina (UFSC). Este hospital escola desenvolve programas de saúde comunitária com grupos especificos de educação para saúde . Com a expectativa de vir formargrupos de hipertensos, justifica-se a realização deste estudo nessa instituição.

A coleta de dados foi realizada nos meses de dezembro de 1991 e janeiro de 1992, de segunda a sexta feira pela manhã e à tarde, período em que a primeira autora dispunha de tempo para esta atividade. A amostragem foi por conveniência, ou seja, aproveitada toda a demanda que aceitou participarda pesquisa eque apresentou o seguinte quadro clínico:P.A. diastólica(PAD)ou mínima maior ou igual a $120 \mathrm{mmHg}$, com queixas ou não (cefaléia, náuses, vômitos, dores, entre outros)semapresentar danos no momento em órgãos considerados alvo. Estesclientesprovavelmenteteriammaiorpossibilidade de participarde programas educativos (gruposde hipertensos). Assim foi possivel entrevistar 24 (vinte equatro) clientes, sendo 14 (quatorze) mulherese 10 (dez)homens. Estasentrevistasocorreram nasalade Pronto Atendimento, após a consulta médica, com um tempo variado de 20 a 40 minutos de duração. O instrumento utilizado para obtenção dosdados constou de um formulário comquestões abertas, norteadorase exploradorasdasituaçãodeestaremcrisehipertensiva, com o objetivo de obteruma noção ampliada desta problemática. Questõessobredadosde identificação (complementaresaosdafichadeatendimentodoSetor deEmergência-HU/UFSC)eclínico-epidemiológicos da crise hipertensiva, foramasmesmas paratodos os clientes, conforme são citados na apresentação descritivadosdados. Este instrumentofoisubmetido a um pré-teste, sendo feitososajustesnecessáriose treinamento da entrevistadora. Alguns dados foram tabulados eapurados, descrevendo-se também em números relativos (percentual). Outros são apresentadosisoladamente, comalgunscomentários equestionamentos.

\section{APRESENTAÇÃO DOS RESULTADOS E COMENTÁRIOS}

\subsection{Caracterização da clientela}

A clientela investigada foi constituída por 14 (58\%) mulheres e 10 (42\%) homens, 23 (96\%) procedentes da Grande Florianópolis. Ressaltase que destes, $12(50 \%)$ possuiam mais de 61 anos de idade, seguidos de $3(12,5 \%)$ entre 51 a 60 anos; 4 (17\%) de 41 a 50 anos; 4 (17\%) de 31 a 40 anos e apenas 1 (4\%) com menos de 30 anos de idade. Todos os participantes eram brancos. Quanto ao estado civil, 19 (80\%) eram casados, 4 $(16 \%)$ eram viúvos e apenas $1(4 \%)$ era solteiro. Ainda, 9 (37,5\%) mal sabiam ler e escrever, 11 $(46 \%)$ tinham o $1^{\circ} \mathrm{grau}$ incompleto e $4(16 \%)$ tinham conseguido chegar ao $2^{\circ} \mathrm{grau}$; no entanto, somente $2(8 \%)$ destes haviam completado o mesmo. Em relação à ocupação, $9(37,5 \%)$ eram aposentados, $6(25 \%)$ do lar, e os $9(37,5 \%)$ restantesestavamligadosàatividade braçal (capoeira, serviços gerais, lavadeira, jardineiro, eletricista, agricultor, operadordexeroxe motorista de ônibus). Quantoàrendafamiliar, pode-seobservarque 14(58\%) 
possuiam renda familiarentre 3 e 5 salários mínimos, enquanto $10(42 \%)$ possuiam rendadeaté 3 mínimos. Em relação ao número de dependentes, 16 (42\%) possuiam mais de 4 dependentes, enquanto que 8 (33\%) de uma três. Igualmente, os quetinham mais dependentesnãoeramosdemaiorrendamensal.

\subsection{Fatores de risco relacionados com a C.H.}

Nas questões relacionadas aos fatores de risco, a obesidade parece ser acompanhante do hipertenso, pois 14 (58\%) destestinhamseu peso acima do considerado ideal* e somente 1 (4\%) tinha seu peso abaixo do teórico ideal. Os demais $9(37,5 \%)$, estavam no limite considerado normal. Com relação à hereditariedade, 20 (84\%) desses clientes referiram que seus pais haviam falecido: $8(34 \%)$ mães e $6(24 \%)$ dos pais, por problemas cardiovasculares (nestes se incluem: derrame cerebral, edema agudo de pulmão e infarto agudo de miocárdio). Importante esclarecer que muitos destes clientes não sabiam exatamente do que seuspaishaviam falecido, podendo inclusive ser mais alto o índice de óbitos conseqüentes à hipertensão.

No que se refere ao uso de bebida alcoólica e uso de cigarros, apenas $2(8 \%)$ referiram beber um copinho de cerveja diariamente e apenas 5 (20\%) referiram ser fumantes; 4 (16\%) destes fumavam, emmédia, meia carteira decigarrosaodia enquanto que $1(4 \%)$ referiu fumar de uma a duas.

Observa-se portanto uma tendência dos indivíduos hipertensos terem na sua história de vida alguns fatores de risco interligados. Confirmase o que dizem a maioria dos autores WOLLF $^{8}$, RIBEIRO ${ }^{6}$, BRUNNER e SUDDARTH ${ }^{3}$, BEYERS e DUDAS ${ }^{1}$ ), que a hipertensão está diretamente vinculada à ocorrência de fatores de risco, como o uso do álcool, fumo, dieta rica em sódio, e ainda fatores como a hereditariedade, obesidade, estress, entre outros.

A realizaçãodealgumtipodelazeré costumede 13 (54\%) dos 24 pacientes entrevistados. Lazerdo tipo caminhadas, visitas a parentes, leituras, idas à praia, pescarias, ouvirrádio, verprogramasdetelevisão, ira

\footnotetext{
* Considerou-se os seguintes critérios para determinar o peso referência (p.r.) ${ }^{5}$

Sexo Estatura média

homens $(H-100)-5 \%$

mulheres $(H-100)-10 \%$
}

festas, a igrejas e passeios diversos. Associado a estes tipo de lazer, alguns clientes também relacionaram, fazer comida, renda, crochê, lavar roupas e outros afazeres domésticos. Parece que lazer e atividade de trabalho estão para alguns intimamente relacionados, e que em determinadas situações até se confundem. Será que o fato de algumas pessoas não terem nenhum tipo de lazer pode este ser um fator estressante? Será que o restrito mundo em que vivem, poderia estar causando crises hipertensivas mais frequentes? Este lazer efetivamente contribuirá para o seu relaxamento? Está havendo relaxamento? Os indivíduos estão conscientes da necessidade de realizar algum tipo de lażere conseqüentemente, reduzir os seus níveis pressóricos?

\section{3 . Motivos da procura da Emergência Hospitalar}

Quando questionados sobre os motivos que os levaram à Emergência Hospitalar, os clientes responderam: desde a simples informação de que estavam com P.A. elevada, até estarem apresentando um conjunto de sinais e sintomas agudos do tipo: dorde cabeça, tontura, edema em membros inferiores, dor no peito, dor no braço esquerdo, dornos rins, vômitos, falta de ar, fraqueza, palidez, "arroxamento", dornas costas, dificuldades para enxergar, desmaio, cólica renal, dificuldade para dormir, tremores, tensão, dificuldades para caminhar, com ameaça de derrame cerebral, com início de edema agudo de pulmão e dificuldades para caminhar. Entretanto, o motivo de maior frequência apontado caracterizou-se pelo aumento excessivo da P.A., associado à cefaléia e tontura . O que se pode constatar através dos níveis de P.A. verificados, é que a máxima ou sistólica variou entre 150 a $300 \mathrm{mmHg}$. Enquanto que, para a minima ou diastólica, os valores variaram entre 120 a $140 \mathrm{mmHg}$. Estes valores são extremamente elevados e perigosos, se considerarmos o que a Organização Mundial de Saúde (OMS) e o Ministério da Saúde ${ }^{5}$ (M.S.) aceitam como níveis pressóricos para um adulto (acima de 20 anos), ou seja: aquele que possui PA igual ou acima de 160 $\mathrm{mmHg}$ para a máxima, ou sistólica, e de $95 \mathrm{mmHg}$ para pressão diastólica ou mínima, é considerado hipertenso. Portanto, estes clientes tinham níveis pressóricos extremamente elevados e corriam risco emergente. 


\subsection{Queixas apresentadas na Emergência}

Naemergência, as principaisqueixasapresentadas pelosclientesforam:tortura(15), cansaço(13), cefaléia (12), taquicardia (12), dor (11), agonia (10), dispnéia (9), edema(7), confusãomental(6), distúrbiomental(5), escotomas (3), torpor(3), edesmaio (1).

\section{5 - Indicação dos motivos que os levaram para desenvolver a Crise Hipertensiva.}

Dos clientes ao serem questionados "sobre $o$ que ocorreu antes da crise", 10 (42\%) deles não sabiam exatamente precisar o motivo, ou algum fato que justificasse o seu desencadeamento. Oito no entanto referiram situações como: "perda de um filho"; "o filho ameaçou suicidar-se"; "o filho o incomodou"; "viu briga entre os filhos"; "viu uma discussão e ficou mal"; "teve uma briga em casa, e a filha quer que vá morar na casa de outros filhos"; "preocupada com a conta bancária"; e "perdeuametadedo salário". Outros relacionaram ainda, situaçס̃es como: "ter se alimentado com dieta muito salgada"; "ter comido em excesso alimentos muito salgados"; "ter se alimentado com carne de porco muito gorda, feijåo e farinha"; "ter deixado de tomar o remédio Moduretic, por estar em falta na farmácia" e ainda um dos respondentes relacionou que "o clima estava demasiadamente quente e desagradável, desencadeando sua crise".

Percebe-se portantoque fatores psicossociais parecem ser essenciaisna ocorrência de aumento nos níveis pressóricos e consequentemente no desencadeamento daC.H. Talvez of fato de serem pessoas muito sensiveis, porsisóvenha justificar, que as turbulências comuns do cotidiano possam ser desencadeadoras de C.H. nesta pessoa.

Selye apud WOLLF ${ }^{8}$, estudando o estress definiu-o como um conjunto de reações de alame face aos estímulos de agressão. No estress, o sistema nervoso está sobrecarregado ao máximo, havendo uma elevação muitas vezes acima do normal na produção de hormônios reguladores da pressão arterial. O estress não é provocado apenas por mudanças de temperatura e doenças, mas principalmente por sobrecargas emocionais do dia a dia, nesse caso chamado de estress psicossocial ${ }^{8}$. O início do estress se dá em função do sfatores causadores e estressantes. $O$ prof. Levy da OMScita alguns fatoresque podem desencadear o estress ou seja "conflitos na vida profissional, na vida matrimonial, na relação entre pais e filhos e nas novas situações que se apresentam na vida das pessoas ${ }^{8}$.

Entretanto, parece que a influência do estress em se transformar em fator de risco para a hipertensão depende principalmente da personalidaded o indivíduo. Segundo BEYERS \& DUDAS ${ }^{1}$ indivíduos do tipo $A$, ou seja, aqueles que se caracterizam por serem extremamente impacientes, terem um senso exagerado de urgência, serem impulsivos e costumarem competir em demasia, são os mais propensos a desenvolverem a hipertensão arterial e doenças cardiovasculares, assim como as crises hipertensivas.

Em nosso trabalho pode-se perceber que, na visão dos clientes, as crises hipertensivas desenvolveram-se em função da ocorrência de conflitos de ordem familiar.

Estes indivíduos já têm na sua história de vida níveis pressóricos elevados, ou seja, são hipertensos, que na maioria das vezes, não se tratam, ou se tratam de forma inadequada; ou ainda desconhecem ser hipertensos. Portanto, em muitas ocasiões, estes indivíduos podem vir a desenvolver a C.H. Nesse momento de C.H., o cliente tende a procurar a emergência hospitalar num estado de ansiedade e angústia e com a expectativa de obteralívio rápido da sintomatologia que o acompanha, tendo como objetivo principal manter-se vivo. Depois de atendido pelo médico e medicado, surge um segund o momento que é o da reflexão sobre sua vida, da forma como a tem conduzido e das possibilidades de mudança. É neste momento de reflexão e busca de soluções, que a enfermeira consegue estabelecer uma relação mais próxima com o cliente, de confiança, solidariedade, de ajuda, procurando de forma conjunta, descobrir novos caminhos, na busca de um estado ideal de saúde, que previna novas crises e que reduza seus niveis pressóricos, trilhando um caminho com novas perspectivas de vida.

\section{CONSIDERAÇÕES FINAIS}

Com este trabalho tivemos a oportunidade de estar em uma emergência hospitalar junto ao indivíduoesuafamíliaqueestavamomentâneamente, 
edeformaintensa, vivenciandoaC.H.Pudemossentir como era visto e tratado este tipo de paciente, e passamosanosquestionarsobre otipodeatendimento recebido porestes, ao nosso ver, muito alémdassuas reaisnecessidades. Emnenhum momento, alémdo tratamento medicamentoso, era oferecidoqualquer outro recurso. Entretanto, há que se considerarquea equipe de saúde não oferecia muito mais do que o tratamentomedicamentoso, não por falta devontade ou interesse, massim talvez, peloexcesso de outras atividadesede pacientesgravíssimosquedemandavam aestesetor.

Podemossentirtambém, queoestresseeconflitos familiares merecem especial atenção quando acompanhamos o tratamento de indivíduos portadores de hipertensão arterial. Acredita-se que a dimensão da atenção da enfermagem deve ultrapassar o foco biológico - corpo na busca de hábitos saudáveis de vida e conseqüentemente, na redução dos níveis pressóricos e da crise hipertensiva.

Portanto, urge que se faça alguma coisa mais poresteindivíduo, uma vez que em muitasocasiões é a emergência hospitalaro local onde ele descobre queé portadorda hipertensão arterial, necessitando portanto de orientações de como tratá-la efetivamente, a fim de evitar a recorrência da crise e outras complicações cardiovasculares.

\section{REFERÊNCIAS BIBLIOGRÁFICAS}

1 - BEYERS, Marjorie e DUDAS, Susan. Enfermagem Médico - Cirúrgica. Tratado de Prática Clínica. Rio de Janeiro: Guanabara Koogan, 1989. 4 volumes.

2 - BRAUNWALD, E. Heart Disease. $3^{\circ}$ ed. Philadelphia: W.B. Saunders Company, 1988.

3 - BRUNNER, Lillian Sholtis \& SUDDARTH, Doris Smith. Tratado de Enfermagem Médico - Cirúrgica. $3^{\circ}$ ed. Rio de Janeiro: Interamericana, 1985.

4 - FERGUNSON, Roger e VLASSES, Peter H. Hypertensive Emergencies and Urgencies. JAMA. v.255, n. 12, p. 1607 - 1613, 'march, 1986.

5 - MINISTÉRIO DA SAÚDE (BRASIL). Normas té c n i c a para o programa nacional de educação e controle de hipertensão arterial. Brasília: Centro de Documentaçăo do Ministério da Saúde, 1988.
6 - RIBEIRO, Artur Beltrame. Hipertensão Arterial. Rio de Janeiro: Marques Saraiva, 1988. 107 p..

7 - VIDT, Donald e GIFFORD, Ray W. Compendium for the treatment of Hipertensive Emergencies. Cleveland Clinic Quartely. v. 51, n. 2, p. 421 - 429, 1984.

8 - WOLFF, Hanns P. Hipertensão arterial: reduçăo da pressăo arterial, prevençăo de derrame cerebral e do infarto do miocárdio. Traduçăo de Cristina Schneider. Rio de Janeiro: Livro Técnico, 1984. 96p.

Recebido para publicaçăo em 17/2/94. 